\title{
Modeling Value on Investment: A Crucial Strategy Adopted by Successful Chronic Condition Management Programs
}

\section{Monica Leslie and Rehan Virani}

Editor's note: In this article, the authors conclude their series on the impact of chronic conditions on our nation. In the April edition of Telehealth and Medicine Today, they explained why chronic conditions will continue to be a focus of cost and utilization issues in our nation's healthcare system and they introduced telehealth as an effective tool to supplement existing Chronic Condition Management (CCM) efforts for increased value creation.

Here, the authors describe how Chronic Condition Management technology creates value on investment (VOI), dwarfing the idea of "returns" on investment (ROI), by using a proprietary model they've created - the Chronic Condition Management Benefits Estimation Tool.

Organizations tend to focus on outcomes attributed directly to their efforts (i.e., fee for service revenue), while missing greater value just under the surface. The Iceberg Analogy (Figure 1) reveals a significant opportunity that lies just below traditional thinking, allowing organizations to improve population health, increase care access, and contain costs of chronic conditions.

Schumacher Clinical Partners Consulting Services assists healthcare organizations in strategizing, as well as designing and implementing programs in the telehealth space. It is our goal to visualize the exponential game-changing nature of telehealth as a resource to lower costs while improving quality and access to healthcare.

In setting down the path to demonstrate that Value on Investment (VOI) is the new Return on Investment (ROI) in the world of telehealth + chronic care, we were 
determined to create a tool to better illustrate this idea-The Chronic Condition Management Benefits Estimation Tool. This article reports on the success of that effort.

\section{Methods}

Over 30 experiences on Chronic Condition Management programs with telehealth were reviewed and are included in this work. Data were evaluated based on the following criteria:

- CCM program type

- Organization type

- Size of the organization

- Technology utilized

A sample of those studies include: Geisinger Health Plan, Lee Memorial Health System, Carolinas Health System, Alterna-Care, University of Rochester, Northern Arizona Health, and Vidant Health. For the purpose of this evaluation, items that generate VOI were introduced into the tool. Then, the impact of telehealth on those items was determined and translated into financial values.

Interviews were conducted with leading CCM programs to better identify and understand the value-generating opportunities resulting from the use of telehealth. From those interviews, all revenue, cost avoidance, and savings opportunities were reviewed to validate those providing clear evidence of benefit.

Revenue-generating items such as the Medicare Chronic Care Management program reimbursing between $\$ 42$ and $\$ 43$ per member per month (PMPM) were identified, as well as the copay from participating Medicare members of \$8 PMPM. Industry resources such as the Centers for Medicare and Medicaid Services, the Centers for Disease Control and Prevention, and Becker's Hospital Review were used to identify benchmark data in order to calculate fiscal advantages. 
Outcomes measured include avoidable readmissions, avoidable index admissions, avoidable Emergency Department visits, reduction in use of ambulance service, and improved CCM resource utilization. Readmissions were defined as an admission to the hospital within 30 days of discharge from an acute care hospital. Index admissions were defined as potential admissions to an acute care hospital that were avoided due to the improved management of chronic conditions through the use of telehealth technologies.

\section{Results}

Criteria that consistently translated into value for the organizations studied included a reduction in costs associated with the following services.

- Avoidable readmissions

- Avoidable index admissions

- Avoidable Emergency Department visits

- Ambulance service utilization

- CCM resource utilization

On average across studies evaluated, the rate of readmissions was reduced $30 \%$, while index admissions were reduced $66 \%$. For other measured parameters, there was a $30 \%$ reduction in emergency visits, an $11 \%$ reduction in the use of ambulance transport, and a $53 \%$ reduction in the use of CCM resources.

\section{Discussion}

The outcomes reported in this analysis focus on major areas for cost containment in healthcare that every hospital must address. In the U.S., hospitals spent $\$ 41.3$ billion between January and November 2011 to treat patients readmitted within 30 days of discharge, according to the Agency for Healthcare Research and Quality (AHRQ). Some 1.8 million readmissions cost the Medicare program $\$ 24$ billion; 600,000 privately insured patient readmissions totaled $\$ 8.1$ billion; and 700,000 Medicaid patient readmissions cost hospitals $\$ 7.6$ billion.

Regarding hospital admissions, an analysis by the Massachusetts Department of 
Health Care Finance and Policy found that the annual cost of Emergency Department overuse in 2005 was approximately $\$ 1$ billion in Massachusetts alone, accounting for 43.3\% of all outpatient Emergency Department charges. In 2011, the American Hospital Association reported that there were more than 129 million Emergency Department visits, an increase of $22 \%$ over the past decade.

Medicare reports that the costs for ambulance transportation was nearly $\$ 6$ billion a year in 2013, up from \$2 billion in 2002.

"Think the ER is Expensive? Look at How Much It Costs to Get There," is the title of an article in The New York Times published in 2013. The Times reminds us, "Thirty years ago ambulance rides were generally provided free of charge, underwritten by taxpayers as a municipal service or provided by volunteers. Today, like the rest of the healthcare system in the United States, most ambulance services operate as businesses and contribute to America's escalating medical bills. Often, they are a high-cost prequel to expensive emergency room visits.

Finally, regarding CCM, about $80 \%$ of older adults have one chronic disease, $68.4 \%$ of Medicare beneficiaries have two or more chronic diseases, and $36.4 \%$ have four or more. Accordingly, it's not surprising that more than two-thirds of all healthcare costs go to treat chronic diseases, while less than $1 \%$ of healthcare dollars are spent on prevention to improve overall health.

Using the CCM tool, a program with a target population of 1,500+ members generates an estimated additional financial value of greater than $\$ 4$ million over 3 years for an organization as a result of implementing telehealth as part of their CCM program. This is a greater than $50 \%$ additional value realized over Chronic Condition Management programs without the availability of telehealth. Factors contributing to this benefit are illustrated in Figure 2. 
The areas of opportunity not represented in part of this analysis are listed below. It is anticipated that they might further contribute value to CCM programs with telehealth.

- Incremental revenue created by increased patient satisfaction

- Increased care plan adherence, disease knowledge and ability to self-manage

- Significantly increased patient quality-of-life

- Reduced acuity level of patients leading to care utilization in lower cost settings and fewer acute episodes

- Shorter length of stays due to reduced acuity levels

- Reduced patient transportation costs

\section{Conclusions}

It is important to note that although not explicitly included in our tool, the landscape for reimbursement of telehealth technology supporting Chronic Condition Management continues to become more favorable. Many commercial and Medicaid programs do provide reimbursement, and those can be easily added to the tool on a case-by-case basis.

In consideration of the changing reimbursement environment at the time these data were collected, the results document that telehealth can generate a significant VOI for CCM programs. However, the mere addition of telehealth should not be expected, on its own, to result in a successful CCM program. Design of the CCM program is critical to its success. Ensuring the program population is engaged and empowered are key to patient participation and use of the telehealth technology. Risk stratification will identify the high-risk patients who will utilize telehealth technologies to monitor and manage their care. Telehealth is therefore a means to enhance a CCM program and drive additional value realization.

Tags: access, admission, ambulance, Becker's Hospital Review, Centers for Disease Control and Prevention, Centers for Medicare and Medicaid Services, chronic care, chronic condition management, emergency department, index admission, modeling, 
readmission, return on investment, $\mathrm{ROI}$, Schumacher, telehealth, value on investment, VOI

Monica Leslie is a Senior Consultant with Schumacher Clinical Partners, with firsthand experience designing and implementing telehealth programs while working in hospital operations.

Rehan Virani is a Consulting Manager with Schumacher Clinical Partners. He specializes in the use of Telehealth to develop new care delivery models. Mr. Virani has many years of experience helping organizations understand and evaluate the VOI as well as implement and operationalize telehealth technologies into daily operations.

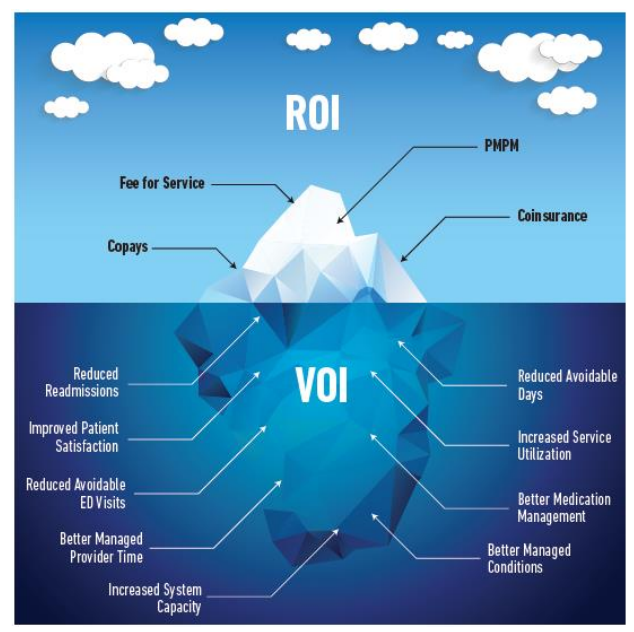

Figure 1. By focusing on what is directly attributed to their efforts (i.e., fee for service revenue), organizations tend to miss significant opportunities to improve population health, increase care access, and contain costs of chronic conditions that lie just below traditional thinking — the Iceberg Analogy. 
1646c. Leslie. Modeling.

https://doi.org/10.30953/tmt.v2.74

Page 7 of 7

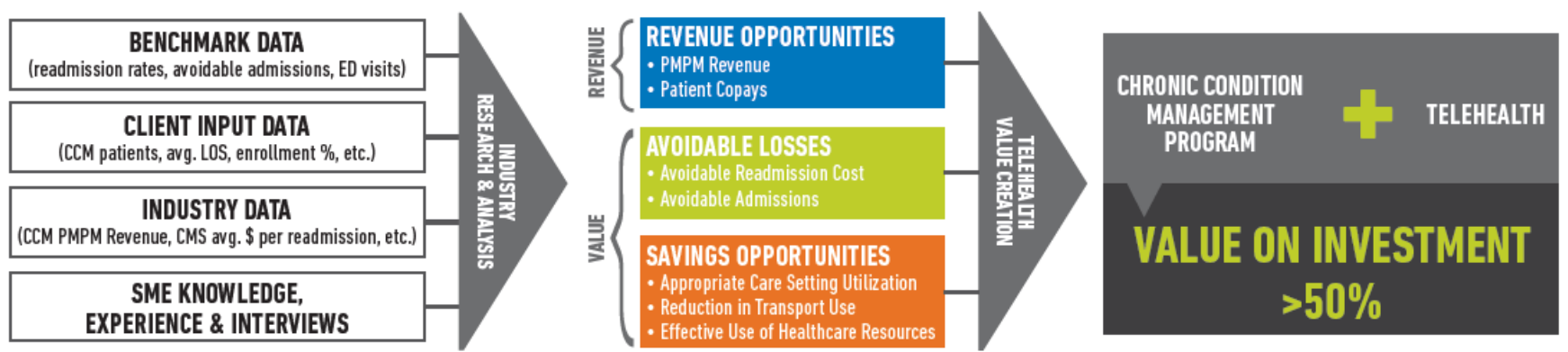

Figure 2. Factors that contribute to VOI for organization as a result of implementing telehealth as part of their CCM program 